\title{
Research on the Development of New-type Urbanization in China
}

\author{
Zhou Hua \\ Yangtze University, Jingzhou, 434023, China
}

Keywords: new-type urbanization; development problems; development path

\begin{abstract}
This research analyzes the current urbanization development direction and the important characteristics through a deep interpretation of the new-type urbanization concept and its development status. Secondly, it focuses on discovering and analyzing the outstanding problems emerged in urbanization process. Finally, it proposes that it is necessary to pursue a three-dimensional new-type urbanization featured with intelligent, green and low-carbon development. Intelligent development represents an important engine of new-type urbanization. Green development is the basic feature while low-carbon development is an important symbol of new-type urbanization.
\end{abstract}

\section{Introduction}

Years after the reform and opening up have witnessed the active development of small towns, and its development has begun to keep abreast with urban development which provides powerful driving force for urbanization. In the new situation of comprehensively promoting urbanization in China, small towns can play a significant role. [1] However, due to its weak foundation and development bottleneck with multi-faceted challenges, the future development still has a long way to go. A major achievement of China's urbanization construction theory is the transformation of development philosophy, and since the scientific outlook on development was put forward in 2003, the guiding ideology of the Chinese urbanization has shifted from the "material-oriented" to "people-centered". [2] The core of the urbanization is the realization of people-centered urbanization. Therefore, we should pursue an intensive, intelligent, green and low carbon urbanization path. Among them, the intensive development is the basic path, intelligent development is the important engine, green development is the basic characteristics and low carbon development is an important symbol of the new-type urbanization.

\section{New-type Urbanization}

New-type urbanization is people-centered and stresses harmonious development integrating the development of industrialization, IT application, urbanization and agricultural modernization. It prioritizes the coordinated development of small towns and cities, medium-sized and large cities and pursues an intensive, intelligent, green and low carbon rural urbanization path characterized with urban culture. [3] The "new" of new-type urbanization is mainly reflected in the change from blind urban sprawl in the past to the emphasis on the connotations development and quality of public services and urban culture, so that farmers have access and opportunities to go to and stay in cities and live a real urban life with dignity. [4] The connotation of new-type urbanization is very rich which can be summarized by the people-oriented urbanization, urbanization of things and sound ecological environment.

\section{The Problems of China's Urbanization Development}

National New Urbanization Planning (2014-2020) points out, "Urbanization is a natural historical process accompanied by industrialization, non-agricultural industries gathering in cities and towns, and rural population concentrating in cities and towns. It is an objective trend of human social development and an important symbol of national modernization.” [5] Urbanization is a huge 
driving force for China's economic growth and also a strategic pivot for China's future development. With a population of 1.3 billion, China still remains a large agricultural country. Although significant progress has been made in urbanization, many problems still exist and the level of development is still lagging behind. Problems such as imbalances and unsustainability in the process of urbanization are still prominent. The serious waste phenomena such as enclosure construction, model projects, semi-urbanization, and urban diseases have all deliver a blow to the healthy development of China's urbanization, resulting in low quality of China's urbanization.

\subsection{Urbanization development lags behind industrial development}

The lagging development of urbanization limits the development of the urbanization-based tertiary industry and weakens the city's population accumulation function, which is not conducive to the optimal allocation of resources. The lagging development of urbanization has weakened the ability of the industrial structure to optimize and upgrade the employment, especially the transfer of rural labor, which will delay the transfer of surplus rural labor. A large number of the idle jobless gather in rural areas will objectively hinder the innovation of agricultural production technology and hurt the agricultural labor productivity. This is one of the important reasons for the overall low efficiency of our national economy.

\subsection{Land urbanization is faster than population urbanization}

The Urban Blue Book published by the Chinese Academy of Social Sciences on July 30, 2013 states that the true total urbanization rate in China is only $42.2 \%$, which is 10.4 percentage points lower than the urbanization rate of the permanent population announced by the National Bureau of Statistics. This indicates that China's urbanization rate is currently overestimated by $10 \%$ according to the standard of citizenization. It not only increases social costs, but also brings security risks and social instability which is not conducive to the social harmony. The contradiction highlighted by the urbanization rate runs counter to the development law of urbanization, which is far from the people's earnest expectations, and also differs from China's original intention of promoting economic development and improving people's living standards through urbanization. Since the various institutional measures for the urbanization of migrant workers have not yet been established and the path for migrant workers to become citizens has not yet been opened, a large number of land-lost farmers' flooding into the cities will inevitably lead to "land urbanization" being faster than "population urbanization". This kind of urban expansion which deviates from the objective law has brought about an irrational industrial layout and a serious shortage of public service supply, and inevitably an "empty city" has emerged. If we continue to promote urbanization according to this model, the rural arable land will continue to be greatly reduced. This will not only endanger the national red line of the arable land and food security, but also will inevitably further aggravate the difficulty of solving the problems of "agriculture, rural areas and farmers".

\subsection{Serious phenomenon of "semi-urbanization"}

"Semi-urbanization" is the immature state of urbanization because it is the land urbanization rather than population urbanization. Its overall characteristic is that employment, labor, income and life in cities but household registration, family numbers, accumulations and roots in rural areas. The rapid development of urbanization in China has been closely linked with "semi-urbanization" in recent years, and "semi-urbanization" brought about some negative influence. First, migrant workers cannot integrate into cities which sows the seeds of social instability. They always do the dirtiest and hardest work but with the least salary. Their low-level consumption requires them to maintain the original living habits and behaviors in modern cities which makes it difficult for them to integrate into the cities. Second, it is not conducive to the development of urban economy with increasing burden on many parties. Migrant workers work outside all year round, leaving women, children and the elderly at home. The elderly lack care, children lack education, and women have to undertake all housework and agricultural work. In the long run, social problems such as "left behind children”, "left behind women” and "empty nesters” will occur and hinder stability of rural areas. 


\section{Build the Development Path of China's New-type Urbanization}

In the new era, we must take New Urbanization Planning (2014-2020) as a guide and take people-oriented urbanization as the core to build new-type towns with intensive, intelligent, green and low-carbon development.

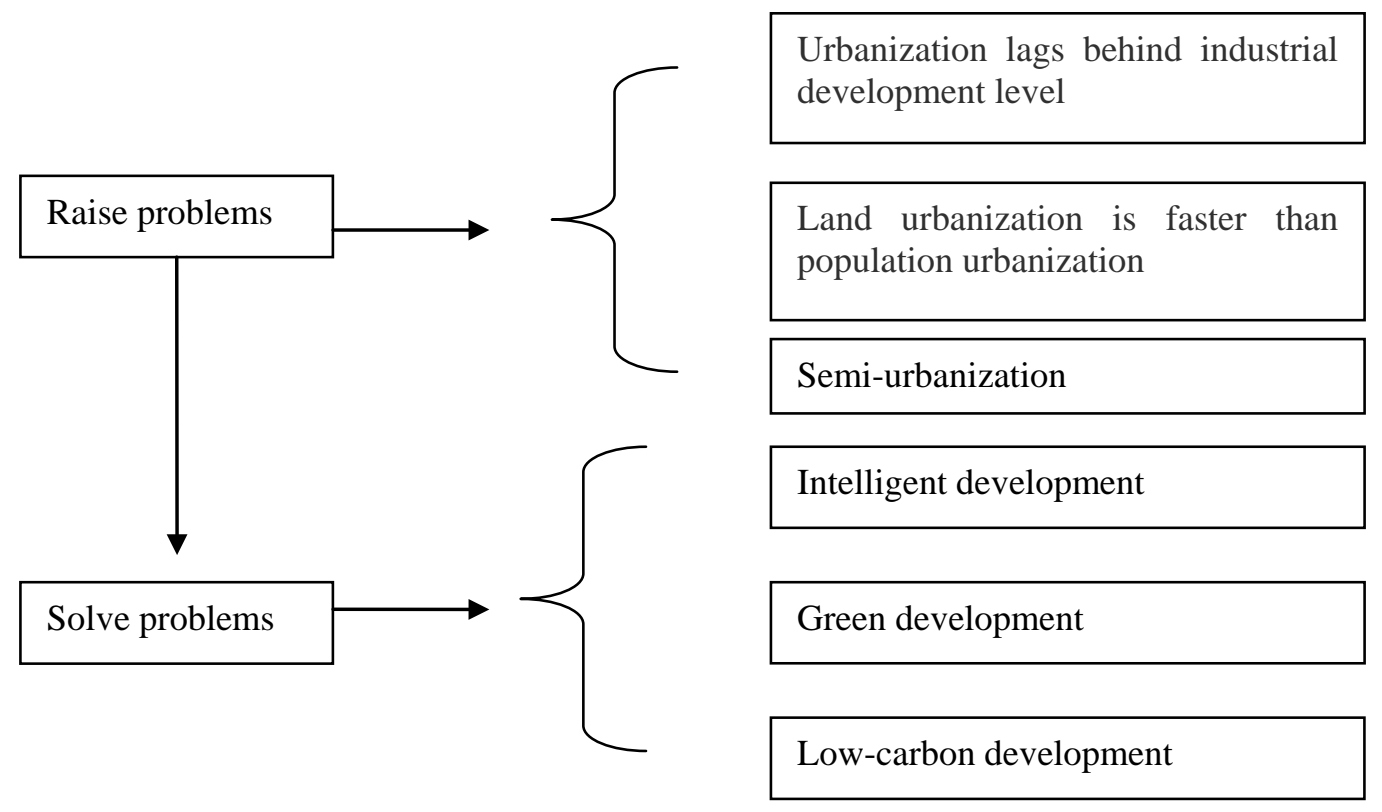

Figure 1 the development path of China's new-type urbanization

\subsection{Intelligent development}

Intelligent development represents an important engine for new-type urbanization. The National New Urbanization Plan (2014-2020) clearly proposes the replacement from the extensive urbanization to intelligent urbanization has become the trend of the future urbanization. As a new engine of China's economic growth, new-type urbanization is a complicated and systematic project. At this stage, efforts are under way to build supporting infrastructure, including smart buildings, rail transit, smart grids and medical information that are closely related to people's lives and activities. In the face of the massive unstructured data generated by the construction of smart cities and towns, big data analysis will greatly improve the "quality" of economic transformation and consumption upgrading. For example, big data technology is used in the medical industry to promote the centralized storage and quick analysis of regional medical information; In the construction of intelligent transportation, comprehensive real-time traffic information monitoring, analysis and intelligent traffic scheduling are realized through big data schemes. In the communications industry, comprehensive recording and analysis of users' communications and Internet surfing behaviors through big data applications to capture users' habits and requirements to provide better and more personalized telecommunication services; To optimize the transportation, inventory and potential shopping requirements of the goods by means of big data analysis in the retail industry; To improve production safety and yield through big data collection and analysis in the production process of manufacturing industry.

\subsection{Green development}

Green development is the basic characteristic of new-type urbanization. There is a growing concern that gray development occupies more green development in industrialization. Therefore, new-type urbanization should base on green development and sound ecological environment. To develop new-type urbanization, we should first take the construction of ecological town as a starting point and make it a breakthrough to solve the contradiction between urbanization and ecological environment. Second, innovate the ecological environment protection system, promote the 
formation of green and emerging urban construction mechanisms, promote the compact and intensive development of cities and towns, thus forming urban planning layouts, industrial structure models and economic development methods not at the expense of resources, energy and ecological environment. Third, establish and improve the assessment system for the ecological environment and incorporate it into the assessment of officials and serve as an important basis for promotion. Finally, establish and improve the system of paid use of resources and ecological compensation system, and implement the most stringent environmental supervision system.

\subsection{Low-carbon development}

Low-carbon development is an important symbol of new-type urbanization. Low-carbon cities and towns adopt low-carbon models with low energy consumption, low emissions, and low pollution under the guidance of the sustainable development philosophy. To build low-carbon cities and towns requires the efforts to develop low-carbon industries, promote cleaner production, improve energy use efficiency, and reduce waste of resources and environmental pollution through technological innovation, energy development, and industrial upgrading. The urban development speed should be controlled within the capacity of resources and environment so as to sustain long-term growth. In terms of specific practices, the government should introduce some preferential policies for low-carbon technological innovation to support the development and innovation of low-carbon industries, but also pay attention to the effectiveness of policies due to the rapid development of low-carbon industries.

\section{Conclusions}

Although great achievements have been made in the construction of urbanization in China, there still remains some problems such as the low quality of urbanization, the slower rate of urbanization than industrial development level and the new phenomena of "urban poor" and "semi-urbanization" in cities. All these pose great difficulties and challenges to the advancement of urbanization. The traditional mode of urbanization is unsustainable for it is at the expense of energy resources and ecological environment. Based on the problems arisen in the development of new cities and towns in China, we must pursue the path of intelligent, green, and low-carbon new-type urbanization, and hope this research have realistic reference value for future urbanization construction.

\section{References}

[1] Probing identity, integration and adaptation: Big questions, little answers[J]. Colleen Ward. International Journal of Intercultural Relations. 2013 (4)

[2] Management, Organizational Justice and Emotional Exhaustion among Chinese Migrant Workers: Evidence from two Manufacturing Firms[J] . Stephen J. Frenkel, MinLi, Simon Lloyd D. Restubog. British Journal of Industrial Relations. 2012 (1)

[3] Mapping form and function in urban areas: An approach based on urban metrics and continuous impervious surface data[J] . Tim Van de Voorde, Wolfgang Jacquet, Frank Canters. Landscape and Urban Planning. 2011 (3)

[4] Spatial Spillovers and Regional Growth: A Cognitive Approach[J] . Roberta Capello. European Planning Studies. 2009 (5)

[5] Characterising the urban environment of UK cities and towns: A template for landscape planning[J] . 